\title{
Dynamic Hierarchical Segmentation of Remote Sensing Images
}

\author{
Giuseppe Scarpa ${ }^{1}$, Giuseppe Masi $^{1}$, Raffaele Gaetano ${ }^{2}$, \\ Luisa Verdoliva $^{1}$, and Giovanni Poggi ${ }^{1}$ \\ 1 DIETI, University Federico II of Naples \\ firstname. lastname@unina.it \\ 2 TELECOM-ParisTech \\ gaetano@telecom-paristech.fr
}

\begin{abstract}
Recursive tree-structured segmentation is a powerful tool to deal with the non-stationary nature of images. By fitting model parameters to each region/class under analysis one can adapt the segmentation algorithm to the local image statistics, thus improving accuracy. However, a single model/segmenter cannot fit regions with wildly different nature, and one should be allowed to select in a suitable library the tool most suited to the local statistics. In this paper, we implement this dynamic segmentation/classification paradigm, using two segmenters, based on spectral and textural properties, respectively, and defining suitable rules for switching model locally. Experiments on remote-sensing mosaics show that the multiple-model dynamic algorithm largely outperforms comparable single-model segmenters.
\end{abstract}

Keywords: Image segmentation, image model, hierarchical segmentation.

\section{Introduction}

Images are inherently non-stationary: most of the information they provide is related to their non-stationary nature. Therefore, simple all-encompassing models cannot capture the complexity of real-world images, and segmentation algorithms based on them are bound to provide poor results. The recent literature testifies a considerable effort [1, 2] towards the development of more complex models and segmentation algorithms for images.

A natural approach is the use of tree-structured hierarchical models and segmentation algorithms, which adapt locally to each region and provide information at different scales of observations of the image. Hierarchical segmentation can be obtained by recursive algorithms, where the image is segmented in a small number of regions, each of which becomes the root of a new local segmentation process, going on until some stopping criterion is met.

A tree-structured segmentation algorithm was proposed in [3], based on local Markov random fields (MRF) models, and hence called TS-MRF. TS-MRF proved to deal quite effectively with image non-stationarity [3, 4], thanks to the opportunity to adapt locally the parameters of the MRF model attached with each node/region. and was also faster than algorithms based on "flat" models. Nonetheless, its flexibility was limited 
to fitting locally the parameters of a given MRF model which could not deal equally well with homogeneous, textured, and strongly structured regions. Therefore, in [5] a dynamical segmentation paradigm with multiple models was proposed, but not implemented, providing just a proof of concept based on supervised experiments. In this work, following the paradigm of [5], we propose and implement a dynamical hierarchical segmentation/classification algorithm, DHC for short, based on two alternative models for homogeneous and textured regions. Here, the major challenge is not the segmentation itself, but the construction of the tree of regions that best describes the image, including thus the selection, at each node, of the model that best fits the region under analysis, and the definition of meaningful growth stopping criteria. To tackle these problems we define a suitable figure, attached with each node/region and each model, called split-gain, which allows us to decide which region to split first, with which model, and when to stop the algorithm.

\section{Background}

In this section we provide a short description of the basic segmentation algorithms, TSMRF and TFR, necessary for understanding the dynamic algorithm proposed here. We assume the reader to have some familiarity with Bayesian segmentation and Markov random fields, referring to specific references [6] for a more thorough treatment.

Tree-Structured-MRF (TS-MRF) is a recursive segmentation algorithm. The whole image of interest, namely the set of sites $\mathcal{S}$ and the corresponding observables $y$ is associated with the root of a tree. Its segmentation divides the set of sites in $K$ disjoint subsets $\left\{\mathcal{S}_{1}, \ldots, \mathcal{S}_{K}\right\}$, each with its subset of observables, associated with the root children. Recursion on the newly generated nodes, together with some suitable stopping conditions, produces a tree of classes and the associated segmentation of the image. An arbitrary number of classes could be considered at each node, but we restrict attention to binary segmentation in order to avoid the challenging task of order selection. At each node, segmentation is carried out according to the MAP criterion

$$
\widehat{x}=\arg \max _{x} p(x \mid y)=\arg \max _{x} p(y \mid x) p(x)
$$

where $p(\cdot)$ indicates probability mass/density function (pmf or pdf) $x$ is the label map, and $\widehat{x}$ is therefore the most probable map given the observables. The label map is modeled as an Ising MRF, where the labels are assumed to be equally likely, only cliques of two 8-connected sites are considered, $c=(i, j)$, and the potentials are defined as

$$
V_{c}\left(x_{i}, x_{j}\right)=\left\{\begin{array}{l}
\beta \text { if } x_{i} \neq x_{j} \\
0 \text { otherwise. }
\end{array}\right.
$$

With this definition, we obtain the global pmf

$$
p(x)=\frac{1}{Z} \exp \left[-\beta N_{E}(x)\right]
$$

where $N_{E}(x)$ is the number of label transition or edges in the map or, under another point of view, the total length of region boundaries in the map. With this prior pmf, label 


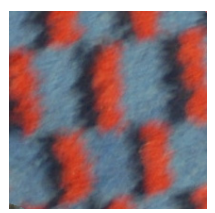

(a)

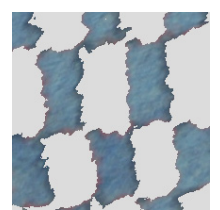

(b)

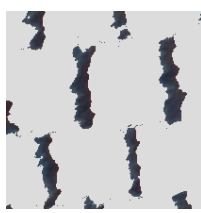

(c)

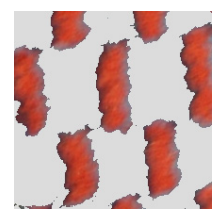

(d)

Fig. 1. An example texture with its three components (clusters of segments)

transitions are penalized, with a strength depending on the edge-penalty parameter $\beta$ : the larger $\beta$ the more regular the final segmentation map.

A key element of TS-MRF is the so called split-gain which, for each node, measures the convenience of actually proceeding with a further split of the associated class. For each leaf node we compute the associated split-gain, and proceed to split the node with the maximum gain, provided it is positive. Therefore, the segmentation tree is shaped by this measure, defined formally and analyzed in depth in the next Section, which also provides the necessary stopping condition.

The Texture fragmentation and reconstruction (TFR) algorithm [7-9] is oriented to the segmentation of textured images. Fig.1 shows a simple texture (a close view of a fabric) together with its decomposition in three basic components, each of which is, in turn, a collection of elementary segments with about the same color, size, and shape. This simple description fits very well a large variety of natural textures, and TFR translates it in three major processing steps

1. color-based segmentation;

2. segment clustering;

3. progressive cluster merging.

The first step detects all elementary connected regions with homogeneous color by means of any conventional region-based or edge-based segmenter. The second steps forms clusters of segments like those shown in Fig.1(b-d), which are the building blocks of textures. To this end, each segment is characterized by a vector of features. These include color, position, size, and shape, but the most discriminating features are related to spatial context, as natural for textures. For example, all black segments of Fig.1(a) have a red segment on their right, a distinctive contextual feature of this specific texture, not easily found in others. Once correctly featured the segment, it is relatively simple to retrieve meaningful clusters by means of standard data analysis techniques. The final step reconstructs the desired textures by progressive pair-wise merging of clusters. A suitable merging gain (called texture score) [9] is defined to decide which texture components should be merged at any step and when to stop the process.

Like TS-MRF, also TFR produces thus a hierarchy of nested segmentation maps. In the latter case, however, the process is a bottom-up merging, starting from the basic clusters of segments to obtain larger and larger textures. However, TFR can be trivially re-engineered to become a top-down process. By forcing the merging process to proceed until only two textures remain, we obtain a binary TFR (B-TFR) algorithm. 
Recursive application of the B-TFR, then, gives rise to a tree-structured top-down segmentation, called recursive TFR (R-TFR) [10], which provides different (and typically better) results than TFR.

\section{Dynamic Hierarchical Classification}

DHC is a recursive algorithm, therefore it can be associated with a tree, where each node corresponds to a region (not necessarily connected) of the image. The root of the tree corresponds to the whole image while the leaves correspond to the elementary segments or classes produced by the segmentation process. We restrict attention to twoclass segmentation and therefore deal with binary trees. A binary tree $T$ is formally identified by a set of nodes $\{t \in T\}$ and their mutual relationships. Except for the root, each node $t$ has one parent $u(t)$, and each internal node has two children $l(t)$ and $r(t)$, with $u[l(t)]=u[r(t)]=t$. In the following, to simplify notation, we will associate nodes with integers, with $t=1$ corresponding to the root, $l(t)=2 t, r(t)=2 t+1$ and $u(t)=\lfloor t / 2\rfloor$.

With each node $t$ in $T$ we associate

- a set of sites $\mathcal{S}^{t} \subseteq \mathcal{S}$, corresponding to a segment of the image;

- a set of observed data $y^{t}=\left\{y_{i}: i \in \mathcal{S}^{t}\right\}$, that is the restriction of $y$ to $\mathcal{S}^{t}$.

A segmentation map $x^{t}$ for node $t$ is a field of labels defined on $\mathcal{S}^{t}$, with $x_{i}^{t} \in\{2 t, 2 t+$ $1\}$. When node $t$ is split according to map $x^{t}$ two new nodes are generated: node $2 t$ with $\mathcal{S}^{2 t}=\left\{i \in \mathcal{S}^{t}: x_{i}^{t}=2 t\right\}$, and $y^{2 t}=\left\{y_{i}: i \in \mathcal{S}^{2 t}\right\}$, and node $2 t+1$ with the associated items defined in a similar way.

Initially, the tree comprises only the root, $T=\{1\}$, which is also its unique leaf. We use the B-MRF segmentation algorithm to compute the segmentation map $x^{\mathrm{S}, 1}$, where the $S$ superscript indicates spectral-based, with split gain $G^{\mathrm{S}, 1}$. Likewise, use the B-TFR segmentation algorithm to compute the segmentation map $x^{\mathrm{T}, 1}$, with split gain $G^{\mathrm{T}, 1}$, where $T$ indicates texture-based. Then we set $G^{1}=\max \left(G^{\mathrm{S}, 1}, G^{\mathrm{T}, 1}\right)$. If $G^{1} \leq 0$ the root is not split, because none of the two segmentation maps provides an improved representation of the data. Otherwise, it is split using the technique with the largest split gain, that is,

$$
x^{1}=\left\{\begin{array}{l}
x^{\mathrm{S}, 1} \text { if } G^{\mathrm{S}, 1}>G^{\mathrm{T}, 1} \\
x^{\mathrm{T}, 1} \text { otherwise }
\end{array}\right.
$$

In the generic step of the algorithm, the leaf with the largest split gain is split using the same logic, provided the gain is positive, otherwise the algorithm stops.

Eventually, it all comes down to the definition of the spectral and textural split gains, which quantify the improvement in representation accuracy, if any, granted by segmentation. In [3] the split gain for TS-MRF is defined as the log likelihood ratio between the competing single-class (no split) and two-class (split) descriptions of the data. In the single-class description of node $t$, there is only one label available, and only one possible label field, with unit probability. The observables are assumed to be independent and identically distributed (i.i.d.) Gaussian with parameters estimated from the data. In the two-class description, instead, the label field is random, $x^{t} \in\{2 t, 2 t+1\}^{N}$, 
and is described by the Ising MRF model of (3), while the observable are conditionally independent, given the label, following two Gaussian laws with different parameters.

Before the log, we therefore have

$$
\exp \left(G^{S, t}\right)=\frac{p\left(x^{t}\right) p\left(y^{t} \mid x^{t}\right)}{p\left(y^{t}\right)}=p\left(x^{t}\right) \cdot \frac{g^{2 t}\left(y^{2 t}\right) g^{2 t+1}\left(y^{2 t+1}\right)}{g^{t}\left(y^{t}\right)}
$$

where $g^{t}(\cdot)$ is the Gaussian distribution that best fits the observables $y^{t}$, with parameters estimated on the data, and $g^{t}\left(y^{t}\right)=\prod_{i \in \mathcal{S}^{t}} g^{t}\left(y_{i}\right)$.

Equation (5) shows clearly the balance among two competing costs/gains. The last fraction makes clear that the two-class hypothesis can provide a better description of the observables as these can be suitably grouped and each group can be explained by a dedicated distribution, $g^{2 t}(\cdot)$ or $g^{2 t+1}(\cdot)$, while in the single-class case a unique distribution $g^{t}(\cdot)$ must explain all data. This obvious description gain is balanced by the cost of describing the grouping itself, represented by $p\left(x^{t}\right)$, which is absent in the single-class case. Therefore, the split gain balances the fitting gain, related to a better description of the data, with the segmentation cost, related to the number of edges $N_{E}\left(x^{t}\right)$ and hence to the map complexity.

We now want to define a split gain for B-TFR segmentation in order to compare the two solutions in a meaningful way. Therefore, we follow the same conceptual path, defining the split gain as a balance between the fitting gain of the data and the segmentation cost. In the case of textures, however, it makes no sense trying to describe data by means of a single Gaussian distribution. When we decide not to split a region, in the TFR framework, it is because we believe it is accurately represented by a single texture, not a single homogeneous field, therefore, its fitting must refer to some kind of mixture distribution. In other words, the region under analysis is considered under two complementary points of view: i) based on spatial properties, it is regarded as a single textured region, ii) based on color, it is divided again in two classes, each represented by its own distribution. Again, we choose the simplest possible mixture to describe data, that is, the mixture of two Gaussians. Therefore, even in the no-split hypothesis, the likelihood of the data is written as

$$
p\left(y^{t}\right)=m\left(y^{t}\right)=\prod_{i \in \tilde{\mathcal{S}}^{2 t}} \tilde{g}^{2 t}\left(y_{i}\right) \prod_{i \in \tilde{\mathcal{S}}^{2 t+1}} \tilde{g}^{2 t+1}\left(y_{i}\right)
$$

where $m\left(y^{t}\right)$ is the best mixture of Gaussians for the data of node $t, \tilde{\mathcal{S}}^{2 t}$ and $\tilde{\mathcal{S}}^{2 t+1}$ are the two segments in which $\mathcal{S}^{t}$ would be divided by the color-oriented clustering map $c^{t}$ (not by the B-TFR map $x^{t}$ ), and $\tilde{g}^{2 t}(\cdot), \tilde{g}^{2 t+1}(\cdot)$ are the associated best fitting Gaussian distributions. Accordingly, in analogy with the former case, the splitting gain for B-TFR is written (again, before the $\log$ ) as

$$
\exp \left(G^{T, t}\right)=\frac{p\left(x^{t}\right) p\left(y^{t} \mid x^{t}\right)}{p\left(y^{t}\right)}=p\left(x^{t}\right) \cdot \frac{m^{2 t}\left(y^{2 t}\right) m^{2 t+1}\left(y^{2 t+1}\right)}{m^{t}\left(y^{t}\right)}
$$

where the map description cost is computed with $x^{t}$ regarded as an Ising MRF. 
Given the estimate of mean vector $\mu$ and covariance matrix $\mathbf{C}$ for each set of observable data, we can write the Fitting cost as

$$
F\left(y^{t}\right)=-\log \left[g^{t}\left(y^{t}\right)\right]=\alpha^{t}+\frac{N^{t}}{2} \log \left|\mathbf{C}^{t}\right|+\sum_{i \in S^{t}} \frac{1}{2}\left(y_{i}-\mu^{t}\right)^{T}\left[\mathbf{C}^{t}\right]^{-1}\left(y_{i}-\mu^{t}\right)
$$

with $\alpha^{t}=N^{t} B \log (2 \pi) / 2$, by which, taking into account also (3), we can write (5) explicitly as

$$
G^{S, t}=\left[F\left(y^{t}\right)-F\left(y^{2 t}\right)-F\left(y^{2 t+1}\right)\right]-\left[\beta^{t} N_{E}\left(x^{S, t}\right)+\log Z^{t}\right]
$$

where the first square brackets accounts for the fitting gain (cost reduction) granted by the split, and the second for the map description cost. Likewise, for a TFR split we can write

$$
G^{T, t}=\left[\sum_{\tau=2 t}^{2 t+1} F\left(\tilde{y}^{\tau}\right)-\sum_{\tau=4 t}^{4 t+3} F\left(\tilde{y}^{\tau}\right)\right]-\left[\beta^{t} N_{E}\left(x^{T, t}\right)+\log Z^{t}\right]
$$

Since all terms scale linearly with the segment dimension, $N^{t}$, we normalize the split gain to it. This has no influence on the comparison between MRF and TFR, but modifies the split order in favor of needy classes, rather than large ones.

A further suitable normalization concerns the data dimensionality. In fact, when $B \gg 1$ (think of hyperspectral images, with hundreds of bands), the regularization term related the map description cost becomes negligible w.r.t. the fitting gain, leading to a sure oversegmentation of the image. Our simple Gaussian model cannot automatically take into account this phenomenon. By normalizing the fitting gain term by the number of bands we increase the probability of stopping, splitting a node only when it is clearly necessary.

Finally, it is well known that the partition function $Z^{t}$ cannot be computed exactly. Some good approximations have been proposed in the literature, especially for the Ising model, but only for the case of rectangular lattices, a constraint that we meet only for the segmentation of the root. Therefore we neglect it altogether, but compare the splitting gain with a small positive threshold, tuned by preliminary experiments, rather than zero.

Eventually, the split gains are defined as

$$
G^{S, t}=\frac{1}{N^{t}}\left\{\frac{1}{B}\left[F\left(y^{t}\right)-F\left(y^{2 t}\right)-F\left(y^{2 t+1}\right)\right]-\beta^{t} N_{E}\left(x^{T, t}\right)\right\}
$$

and

$$
G^{T, t}=\frac{1}{N^{t}}\left\{\frac{1}{B}\left[\sum_{\tau=2 t}^{2 t+1} F\left(\tilde{y}^{\tau}\right)-\sum_{\tau=4 t}^{4 t+3} F\left(\tilde{y}^{\tau}\right)\right]-\beta^{t} N_{E}\left(x^{T, t}\right)\right\}
$$

\section{Experiments and Discussion}

To test the proposed algorithm, we carry out numerical experiments on multispectral remote-sensing images. Segmentation is especially relevant in this context not only as 

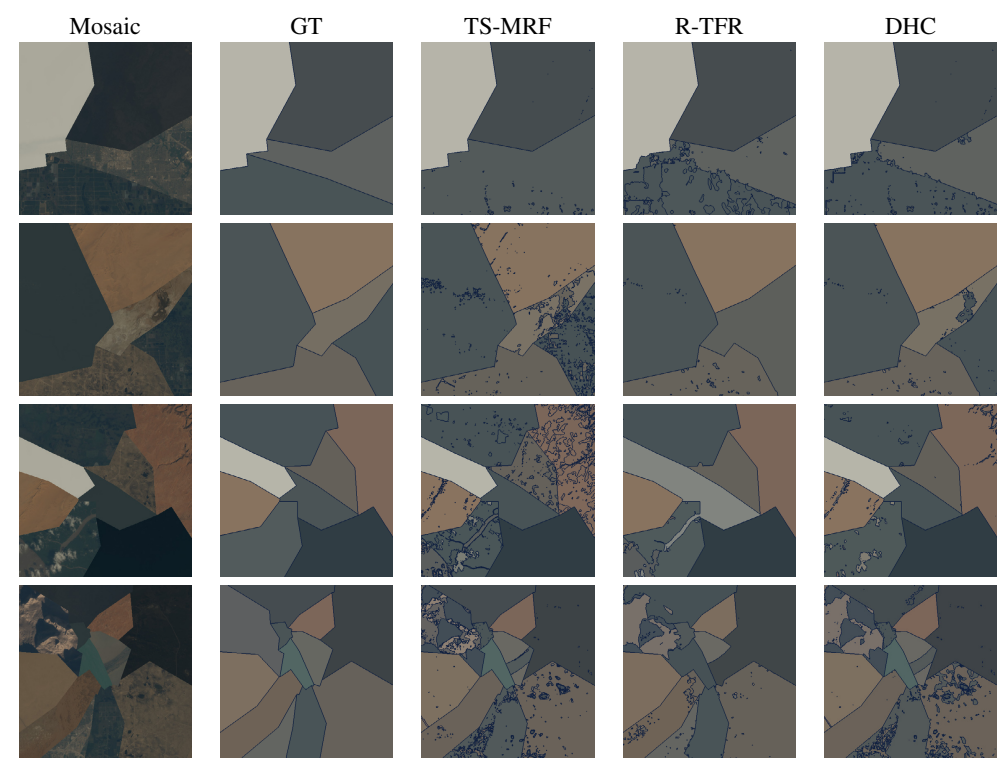

Fig. 2. Results on Prague benchmark. Left to right: mosaic, ground truth, TS-MRF, R-TFR, DHC.

an help for image analysis, but also in related applications, such as content-based image retrieval [11], or region-based image compression [12, 13] and denoising [14]. Objectivity and reproducibility of results are guaranteed by resorting to the publicly available Prague remote-sensing segmentation benchmark [15], which provides a number of multispectral mosaics with attached ground-truth, with many challenging cases. Results are compared under a large number of criteria with those of TS-MRF and R-TFR under two conditions $i$ ) fixed number of nodes $i$ ) best number of nodes (with maximum correct segmentation). In any case, we keep the tree structure provided by the algorithm, with no user intervention.

Fig. 2 shows some of the mosaics used in the experiments together with the associated ground truth and with the best segmentation provided by TS-MRF, R-TFR and DHC, respectively. Visual inspection testifies the superiority of the dynamic model approach: TS-MRF captures well the image structure, most of the times, but produces an obvious fragmentation of regions; on the other hand, TFR provides a more compact map, but happens to erroneously merge some segments. Most of these problems are overcome by DHC. Numerical results in Table 1, referring to a total of 20 mosaics, fully confirm this impression, with DHC almost always the best technique both for the fixed-number and best-number cases. In particular, DHC provides a 5 percent points improvement in overall Correct Segmentation (CS) w.r.t. the best fixed-model reference (R-TFR).

To gain insight into the reasons of such an improvement consider the segmentation tree of Fig.3, obtained by using exclusively the TS-MRF technique. In the box associated with each node we report the TS-MRF split gain (spectral gain, SG) and also, in smaller font, the TFR split gain (or texture gain, TG), which however is not taken into 
Table 1. Numerical results on Prague benchmark for DHC, R-TFR and TS-MRF (with manual selection of the number of classes); (Benchmark criteria: $\mathrm{CS}=$ correct segmentation; OS = oversegmentation; $\mathrm{US}=$ under-segmentation; $\mathrm{ME}=$ missed error; $\mathrm{NE}=$ noise error; $\mathrm{O}=$ omission error; $\mathrm{C}=$ commission error; $\mathrm{CA}=$ class accuracy; $\mathrm{CO}=$ recall - correct assignment; $\mathrm{CC}=$ precision - object accuracy; I. = type I error; II. = type II error; EA = mean class accuracy estimate; $\mathrm{MS}=$ mapping score; $\mathrm{RM}$ = root mean square proportion estimation error; $\mathrm{CI}=$ comparison index; GCE = Global Consistency Error; $\mathrm{LCE}=$ Local Consistency Error; $\mathrm{dD}=$ Van Dongen metric; $\mathrm{dM}=$ Mirkin metric; $\mathrm{dVI}=$ variation of information).

\begin{tabular}{|c|c|c|c|c|c|c|}
\hline & \multicolumn{6}{|c|}{ Benchmark - ALI } \\
\hline & $\begin{array}{c}\text { DHC } \\
\text { Optimal \#f of } \\
\text { classes }\end{array}$ & $\begin{array}{c}\text { R-TFR } \\
\text { Optimal \# of } \\
\text { classes }\end{array}$ & $\begin{array}{l}\text { TS-MRF } \\
\text { Optimal \# of } \\
\text { classes }\end{array}$ & $\begin{array}{l}\text { DHC } \\
\text { Given \# of } \\
\text { classes }\end{array}$ & $\begin{array}{l}\text { R-TFR } \\
\text { Given \# of } \\
\text { classes }\end{array}$ & $\begin{array}{l}\text { TS-MRF } \\
\text { Given \# of } \\
\text { classes }\end{array}$ \\
\hline$C S$ & 84.60 & 78.45 & 66.45 & 75.05 & 70.33 & 55.49 \\
\hline$\downarrow O S$ & 6.78 & 10.39 & 9.34 & 7.56 & 12.95 & 7.58 \\
\hline$\downarrow U S$ & 8.73 & 14.33 & 12.09 & 16.77 & 17.45 & 20.44 \\
\hline$\downarrow M E$ & 4.10 & 2.90 & 14.26 & 4.07 & 8.28 & 15.33 \\
\hline$\downarrow N E$ & & 1.28 & 14.77 & 4.62 & 6.85 & 14.57 \\
\hline$\downarrow O$ & 9 & 1.22 & 4.39 & 3.24 & 1.07 & 8.15 \\
\hline$\downarrow C$ & & 3.11 & 6.07 & 0.52 & 4.78 & 6.40 \\
\hline$\uparrow C A$ & 89.69 & 84.74 & 80.82 & 83.36 & 80.52 & 73.11 \\
\hline$\uparrow C O$ & 92.80 & 89.72 & 86.98 & 88.64 & 86.42 & 81.33 \\
\hline$\uparrow C C$ & 92.78 & 88.98 & 87.88 & 87.19 & 88.30 & 81.47 \\
\hline$\downarrow l$. & 20 & 10.28 & 13.02 & 11.36 & 13.58 & 18.67 \\
\hline$\downarrow / l$. & & 1.25 & 2.64 & 2.25 & 1.98 & 4.23 \\
\hline$\uparrow E A$ & 92.29 & 88.37 & 86.27 & 86.73 & 85.58 & 79.27 \\
\hline$\uparrow M S$ & 89.59 & 85.45 & 81.60 & 82.95 & 79.63 & 72.00 \\
\hline$\downarrow R M$ & & 3.46 & 3.66 & 3.42 & 4.23 & 5.40 \\
\hline$\uparrow \mathrm{Cl}$ & 92.53 & 88.84 & 86.83 & 87.24 & 86.44 & 80.27 \\
\hline$\downarrow G C E$ & 4.38 & 4.34 & 10.26 & 5.74 & 8.45 & 12.31 \\
\hline$\downarrow \angle C E$ & 67 & 2.25 & 6.72 & 2.80 & 2.84 & 6.52 \\
\hline$\downarrow d D$ & & 6.20 & 9.07 & 6.70 & 8.42 & 11.79 \\
\hline$\downarrow d M$ & & 3.06 & 5.66 & 4.33 & 4.60 & 8.38 \\
\hline$\downarrow d V I$ & 14.51 & 14.43 & 14.77 & 14.30 & 14.57 & 14.44 \\
\hline
\end{tabular}

account for the segmentation. The four-class mosaic associated with the root, node 1 , is split along the red line with a large gain, $S G=0.63$. Both children nodes have a positive split gain, close to zero the left one, quite large the right one, which is therefore split first, generating nodes 6 and 7. Now look at node 6: although two different regions are clearly recognizable, the spectral gain is negative, because both regions are internally textured, and hence poorly represented by a single Gaussian fit. This node will no longer be split, with an unrecoverable undersegmentation. Going on we obtain only a wrong split of node 7 and possibly (depending on the decision threshold) of other nodes. In this case, instead, TFR would have split node 3 along the yellow line, correctly separating the two regions, with a relatively large texture gain $\mathrm{TG}=0.16$.

Consider now the symmetric situation of Fig.4, where only the TFR segmenter is used. After splitting nodes 1, 3, and 2, in this order, along the red lines, the algorithm either stops, if the decision threshold exceeds 0.07 , or splits unduly node 5 which corresponds to a homogeneous region. Node 6 is not split because the relatively large gain, $\mathrm{TG}=0.10$, is obtained with a wrong split (not shown) which singles out an exceedingly 


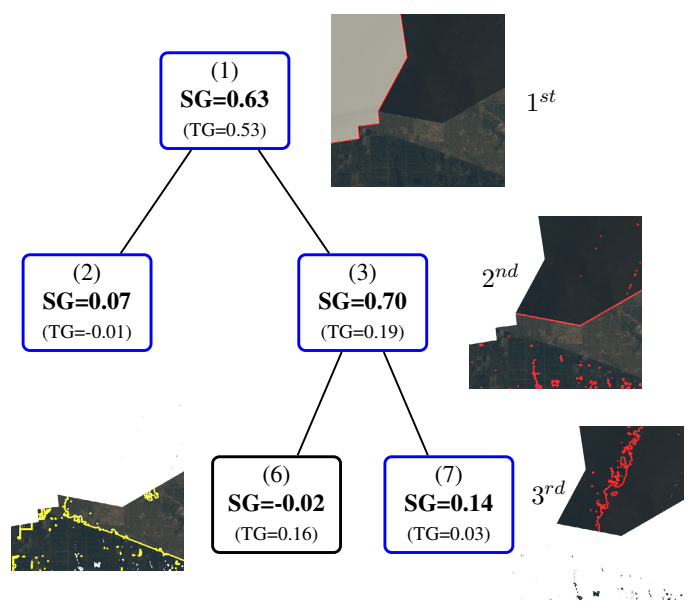

Fig. 3. Tree structured segmentation of mosaic \# 2 by TS-MRF

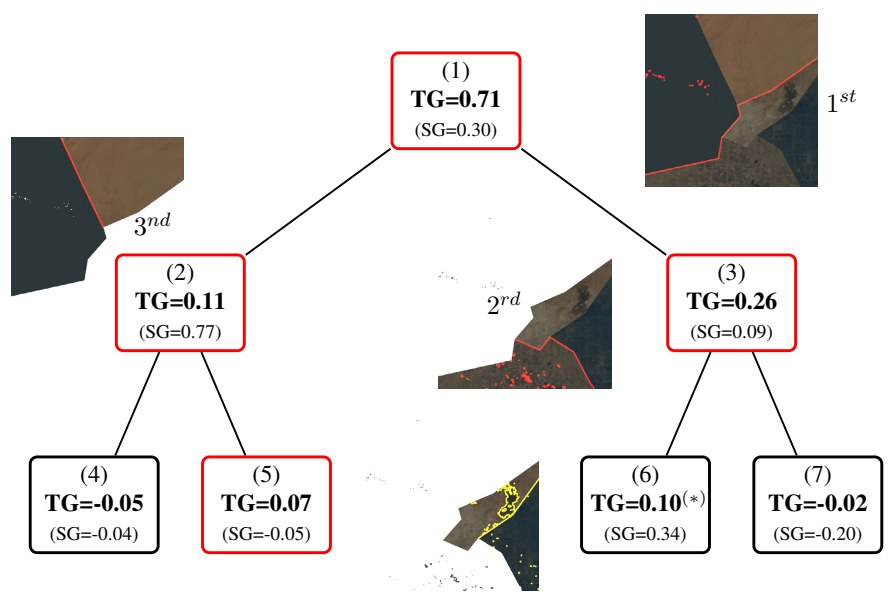

Fig. 4. Tree structured segmentation of mosaic \# 2 by R-TFR

small region, and is hence rejected (marked with a*). The problem is that the two class region associated with node 6 is already well represented by a mixture of Gaussians, making a further textural split not convenient. For the same node, however, the spectral gain would be much larger, corresponding to the correct separations of the two large component regions along the yellow line.

In both cases, the use of the competing models would definitely improve the segmentation path and the final result. These results, however preliminary and limited, clearly show the potential of the dynamic hierarchical classification paradigm. Significant further improvements can be expected by including other image models and segmentation engines. The problem remains of how to define suitable metrics to switch between one model and the other. 


\section{References}

1. Benboudjema, D., Pieczynski, W.: Unsupervised Statistical Segmentation of Nonstationary Images Using Triplet Markov Fields. IEEE Trans. on Pattern Analysis and Machine Intelligence 29, 1367-1378 (2007)

2. Liu, G., Qin, Q., Mei, T., Xie, W., Wang, L.: Supervised Image Segmentation Based on TreeStructured MRF Model in Wavelet Domain. IEEE Geoscience and Remote Sensing Letters 6 , 850-854 (2009)

3. D’Elia, C., Poggi, G., Scarpa, G.: A tree-structured Markov random field model for Bayesian image segmentation. IEEE Trans. on Image Processing 12, 1259-1273 (2003)

4. D'Elia, C., Marrocco, C., Molinara, M., Poggi, G., Scarpa, G., Tortorella, F.: Detection of microcalcifications clusters in mammograms through TS-MRF segmentation and SVM-based classification. In: The 17th International Conference on Pattern Recognition, ICPR 2004, vol. 3, pp. 742-745 (2004)

5. Masi, G., Gaetano, R., Scarpa, G., Poggi, G.: Dynamic segmentation for image information mining. In: IEEE International Geoscience and Remote Sensing Symposium, IGARSS 2010, pp. 1992-1995 (July 2010)

6. Li, S.Z.: Markov random field modeling in image analysis. Springer (2001)

7. Scarpa, G., Haindl, M.: Unsupervised texture segmentation by spectral-spatial-independent clustering. In: The 18th International Conference on Pattern Recognition, ICPR 2006, vol. 2 , pp. 151-154 (August 2006)

8. Scarpa, G., Haindl, M., Zerubia, J.: A Hierarchical Finite-State Model for Texture Segmentation. In: IEEE International Conference on Acoustics, Speech and Signal Processing, ICASSP 2007, vol. 1, pp. I-1209-I-1212 (2007)

9. Gaetano, R., Scarpa, G., Poggi, G.: Hierarchical texture-based segmentation of multiresolution remote-sensing images. IEEE Trans. on Geoscience and Remote Sensing 47, 2129-2141 (2009)

10. Gaetano, R., Scarpa, G., Poggi, G.: Recursive Texture Fragmentation and Reconstruction segmentation algorithm applied to VHR images. In: IEEE International Geoscience and Remote Sensing Symposium, IGARSS 2009, pp. IV-101-104 (2009)

11. Li, Y., Bretschneider, T.R.: Semantic-Sensitive Satellite Image Retrieval. IEEE Trans. on Geoscience and Remote Sensing 45, 853-860 (2007)

12. Cagnazzo, M., Poggi, G., Verdoliva, L.: Region-based transform coding of multispectral images. IEEE Trans. on Image Processing 16, 2916-2926 (2007)

13. Cagnazzo, M., Parrilli, S., Poggi, G., Verdoliva, L.: Improved class-based coding of multispectral images with shape-adaptive wavelet transform. IEEE Geoscience and Remote Sensing Letters 4, 566-570 (2009)

14. Parrilli, S., Poderico, M., Angelino, C.V., Scarpa, G., Verdoliva, L.: A nonlocal approach for SAR image denoising. In: IEEE International Geoscience and Remote Sensing Symposium, IGARSS 2010, pp. 726-729 (2010)

15. Mikes, S., Haindl, M., Scarpa, G.: Remote sensing segmentation benchmark. In: The 7th IAPR International Workshop on Pattern Recognition in Remote Sensing, PRRS 2012, Tsukuba Science City, Japan (November 2012) 